\title{
Comparison of Memory Function and MMPI-2 Profile between Post-traumatic Stress Disorder and Adjustment Disorder after a Traffic Accident
}

\author{
Sung-Man Bae ${ }^{1}$, Myoung-Ho Hyun ${ }^{3}$, Seung-Hwan Lee ${ }^{1,2}$ \\ ${ }^{1}$ Department of Psychiatry, Inje University Ilsan Paik Hospital, ${ }^{2}$ Clinical Emotion and Cognition Research Laboratory, Goyang, ${ }^{3}$ Department \\ of Psychology, Chung-Ang University, Seoul, Korea
}

\begin{abstract}
Objective: Differential diagnosis between post-traumatic stress disorder (PTSD) and adjustment disorder (AD) is rather difficult, but very important to the assignment of appropriate treatment and prognosis. This study investigated methods to differentiate PTSD and AD.

Methods: Twenty-five people with PTSD and 24 people with AD were recruited. Memory tests, the Minnesota Multiphasic Personality Inventory 2 (MMPI-2), and Beck's Depression Inventory were administered.

Results: There were significant decreases in immediate verbal recall and delayed verbal recognition in the participants with PTSD. The reduced memory functions of participants with PTSD were significantly influenced by depressive symptoms. Hypochondriasis, hysteria, psychopathic deviate, paranoia, schizophrenia, post-traumatic stress disorder scale of MMPI-2 classified significantly PTSD and AD group.

Conclusion: Our results suggest that verbal memory assessments and the MMPI-2 could be useful for discriminating between PTSD and AD.
\end{abstract}

KEY WORDS: Diagnosis, Differential; Post-traumatic stress disorders; Adjustment disorders; Memory deficits; MMPI-2.

\section{INTRODUCTION}

Post-traumatic stress disorder (PTSD) can be diagnosed only if the accident (trauma) is severe and psychological distress is extreme. In contrast, adjustment disorder (AD) can be diagnosed in patients who did not meet the diagnostic criteria of PTSD or other Axis I diagnoses of Diagnostic and Statistical Manual of Mental Disorders, fourth edition (DSM-IV) after trauma. ${ }^{1)}$

To differentiate PTSD from other conditions, researchers have focused on cognitive appraisals (e.g., perceived threat) and coping strategy variables that have been recognized as significant predictive factors of PTSD. ${ }^{2-10)}$ However, cognitive and neurocognitive factors, such as intelligence and memory, have received relatively little attention despite the importance of these factors in the differentiation of PTSD from other conditions.

\footnotetext{
Received: October 15, 2013 / Revised: November 11, 2013

Accepted: November 13, 2013

Address for correspondence: Seung-Hwan Lee, MD, PhD, Department of Psychiatry, Inje University Ilsan Paik Hospital, 170 Juhwa-ro, Ilsanseo-gu, Goyang 411-706, Korea Tel: +82-31-910-7260, Fax: +82-31-910-7268 E-mail: Ishpss@paik.ac.kr
}

Previous studies have reported that memory impairment is associated with PTSD symptoms. ${ }^{11-14)}$ Several studies have shown a significant difference in verbal memory ability between people with PTSD and control groups (health group, major depressive disorder and anxiety disorder). ${ }^{15-17)}$ But, to date, there has been minimal research regarding comparison of cognitive function between PTSD and AD. Differential diagnosis between PTSD and AD is rather difficult, but very important to the assignment of appropriate treatment and prognosis. And successful differential diagnoses could also help solve problems for both insurance companies and victims.

Johnsen and Asbjørnsen ${ }^{18)}$ administered the California Verbal Learning Test to examine differences in encoding strategies and verbal memory in individuals exposed to war with and without PTSD (including adjustment disorder). In their study, the serial cluster score was significantly lower, and intrusive errors were more frequent in the group of individuals with PTSD. Geuze et al. ${ }^{16)}$ compared verbal and visual memory between 25 Dutch veterans with PTSD and 25 veterans without PTSD (including adjustment disorder). There was a significant differences in verbal and visual memory testing.

() This is an Open-Access article distributed under the terms of the Creative Commons Attribution Non-Commercial License (http://creativecommons.org/licenses/by-nc/3.0) which permits unrestricted non-commercial use, distribution, and reproduction in any medium, provided the original work is properly cited. 
Table 1. Demographic characteristics and estimated intelligence quotient (IQ) for PTSD and AD group

\begin{tabular}{lccc}
\hline & PTSD $(n=25)$ & AD $(n=24)$ & t-value or chi-squares \\
\hline Gender & & & 0.88 \\
$\quad$ Male & 13 & 13 & 0.25 \\
Female & 12 & 11 & -1.43 \\
Age (year) & $45.20 \pm 13.19$ & $44.21 \pm 14.25$ & -1.94 \\
Education (year) & $9.88 \pm 4.36$ & $11.33 \pm 2.58$ & 1.00 \\
Estimated $1 Q$ & $97.12 \pm 13.31$ & $103.13 \pm 7.13$ & 0.42 \\
Time since accident (day) & $108.68 \pm 99.66$ & $84.38 \pm 66.91$ & \\
Driving status & 11 & 8 & 8 \\
$\quad$ Driver & 5 & 5 & 3 \\
$\quad$ Pedestrian & 8 & 1 & \\
$\quad$ Passenger & 1 & 3 & \\
Etc. &
\end{tabular}

Values are presented as mean \pm standard deviation or number only.

PTSD, post-traumatic stress disorder; AD, adjustment disorder.

It is generally accepted that comorbidity of PTSD and depression is high, and that depressive symptoms can influence mental processing, organizing of information, and memory. ${ }^{19)}$ Memory impairment can be also related to the depressive symptoms of people with PTSD. ${ }^{15,18,20)}$ Thus, to verify the specific effects of PTSD symptoms on memory impairment, it is necessary to control for depressive symptoms. $^{21)}$

Many studies have used the Minnesota Multiphasic Personality Inventory 2 (MMPI-2) to differentiate between people with or without PTSD. ${ }^{22-24)}$ Related studies have suggested that the depression (D), psychasthenia (PT), and schizophrenia (SC) scales of the MMPI-2 are important for the diagnosis of PTSD. ${ }^{25-27)}$ Some studies have focused on the D-SC and PT-SC code types. ${ }^{28-31)}$ The Keane PTSD scale (PK) has also been recognized as a good assessment tool for the differentiation of groups of people with and without PTSD. ${ }^{32,33)}$ However, little is known about which scales of the MMPI-2 are the most useful for differentiating PTSD and AD.

Previous studies ${ }^{16,18)}$ have indicated that tests of memory ability and the MMPI-2 may be useful for differentiating groups of people with and without PTSD (including adjustment disorder); however, almost all of these studies were conducted with veterans, which limits the generalization of results to victims of other types of trauma. Thus, we investigated the usefulness of these assessments in the differentiation of PTSD and AD after trauma from traffic accidents by using a cross-sectional design. We hypothesized that PTSD and AD with traffic accident could be differentiated clinically through memory tests and the MMPI-2 in this study.

\section{METHODS}

\section{Participants}

Twenty-five people with PSTD and 24 people with AD were recruited from 98 eligible individuals. The groups were matched for age, gender, education, estimated intelligence, and elapsed time since the vehicle accident. The mean age of all subjects was 44.71 years old (standard deviation [SD], 13.58), and the mean elapsed time since the accident was 96.78 days (SD, 85.21). Subjects received no economic compensation for their participation. Demographic characteristics of both groups are presented in Table 1 . This study was approved by the institutional review board of Inje University Ilsan Paik Hospital (Goyang, Korea), and all subjects gave informed consent.

\section{Measures}

Korean-Wechsler Adult Intelligence Scale (K-WAIS)

To estimate intelligence quotient (IQ), we utilized an abbreviated form of the K-WAIS, which consists of four subtests (vocabulary, arithmetic, block design, and picture arrangement) and standardized in the article of Yeom et $a l .{ }^{34)}$ We excluded patients whose IQ was below 70. The correlation between the abbreviated form of the WAIS and the full IQ score has been reported to be as high as $0.92-0.94 .^{35,36)}$

\section{Rey-Kim memory test ${ }^{37)}$}

This test consists of two subtests: the Auditory Verbal Learning Test (AVLT) for verbal memory ability and the Complex Figure Test (CFT) for visual memory ability. The AVLT includes immediate verbal memory, delayed verbal recall, and delayed verbal recognition. CFT in- 
cludes immediate figure recall and delayed figure recall.

\section{MMPI-2}

The MMPI- 2 was developed by Hathaway and McKinley, ${ }^{38)}$ and the Korean version was standardized by Han and Hong. ${ }^{39)}$ It consists of 567 questions and has more content and supplementary scales than the MMPI. The MMPI-2 consists of ten clinical subtests: hypochondriasis (HS), depression (D), hysteria (HY), psychopathic deviate (PD), masculinity-femininity (MF), paranoid (PA), psychasthenia (PT), schizophrenia (SC), hypomania (MA), and social introversion (SI). In this study, the reliability of the MMPI-2 averaged 0.86 .

\section{Beck Depression Inventory (BDI)}

The BDI is a self-report instrument used to measure depressive symptoms. ${ }^{40)}$ It consists of 21 questions, which include emotional, cognitive, motivational, and behavioral symptoms of depression. A high total score represents more severe depression. Each question is rated from 0 to 3 points, and the total score ranges from 0 to 63 points. This study used the BDI standardized by Rhee $e t a l^{41)}$

\section{Procedure}

Patients who were at least 18 years old and had been involved in a vehicle accident within one year (the traffic ac- cident occurred between one month and one year prior to the study) were screened for inclusion in this study. Licensed clinical psychologists interviewed patients, and the Structured Clinical Interview for DSM-IV (SCID) ${ }^{39)}$ was used to diagnose PTSD and AD.

Subjects who had brain injuries, diagnoses of intellectual disability, dementia, amnestic disorder, cognitive disorder, personality change due to head trauma, and other psychiatric disorder except for PTSD or AD were excluded. Final diagnosis was determined through consensus at a meeting of the interviewers. Inter-rater reliability for the final diagnosis was approximately $90 \%$. Next to, 4 licensed clinical psychologists administrated K-WAIS, Rey-Kim memory test. Finally, all subjects reported MMPI-2 and BDI.

\section{Statistical Analyses}

For analysis of demographic characteristics, independent $t$-tests and chi-square tests were conducted. We also conducted independent t-tests to compare verbal and visual memory scores, the scores on the MMPI-2, and the BDI scores across participants with PTSD and participants with AD. Pearson correlation analysis was used to identify relationships between depressive symptoms, PTSD symptoms, and neuropsychological functioning. Finally, to explore the unique effect of PTSD symptoms on memory

Table 2. T-test results between PTSD and AD for the memory test, BDI, and MMPI-2

\begin{tabular}{|c|c|c|c|c|}
\hline & PTSD $(n=25)$ & $A D(n=24)$ & Effect size & $t$-value \\
\hline Verbal immediate memory & $7.12 \pm 1.90$ & $8.95 \pm 2.04$ & 0.11 & $-3.18^{\star \star}$ \\
\hline Verbal delayed recall & $7.32 \pm 2.36$ & $8.64 \pm 2.15$ & 0.04 & -1.99 \\
\hline Verbal delayed recognition & $6.56 \pm 3.12$ & $9.41 \pm 2.15$ & 0.14 & $-3.59^{\star \star}$ \\
\hline Figure immediate recall & $8.20 \pm 3.04$ & $8.50 \pm 2.20$ & 0.00 & -0.38 \\
\hline Figure delayed recall & $7.84 \pm 2.84$ & $8.18 \pm 1.92$ & 0.00 & -0.48 \\
\hline \multicolumn{5}{|l|}{ MMPI-2 scale } \\
\hline$L$ & $47.08 \pm 9.29$ & $48.33 \pm 10.55$ & 0.00 & -0.44 \\
\hline K & $47.64 \pm 11.08$ & $47.46 \pm 8.41$ & 0.00 & -0.06 \\
\hline$S$ & $45.52 \pm 11.77$ & $48.22 \pm 9.43$ & 0.02 & -0.87 \\
\hline HS & $62.40 \pm 10.06$ & $56.00 \pm 11.32$ & 0.04 & $2.09^{*}$ \\
\hline $\mathrm{D}$ & $65.32 \pm 13.35$ & $59.38 \pm 13.31$ & 0.06 & 1.56 \\
\hline HY & $64.28 \pm 12.00$ & $54.58 \pm 9.56$ & 0.09 & $3.12^{* *}$ \\
\hline PD & $63.52 \pm 11.00$ & $54.26 \pm 12.95$ & 0.18 & $2.68^{*}$ \\
\hline MF & $51.56 \pm 7.37$ & $46.83 \pm 8.98$ & 0.00 & 2.02 \\
\hline PA & $62.08 \pm 14.14$ & $54.00 \pm 13.49$ & 0.08 & $2.05^{\star}$ \\
\hline PT & $65.96 \pm 15.01$ & $59.17 \pm 13.51$ & 0.08 & 1.66 \\
\hline SC & $64.48 \pm 13.76$ & $55.08 \pm 13.20$ & 0.09 & $2.44^{*}$ \\
\hline MA & $54.12 \pm 12.69$ & $49.75 \pm 9.20$ & 0.01 & 1.38 \\
\hline $\mathrm{SI}$ & $57.56 \pm 13.50$ & $55.04 \pm 14.38$ & 0.04 & 0.63 \\
\hline Keane PTSD scale & $65.16 \pm 14.53$ & $56.21 \pm 13.95$ & 0.10 & $2.20^{*}$ \\
\hline $\mathrm{BDI}$ & $34.56 \pm 11.45$ & $21.35 \pm 7.37$ & 0.22 & $4.79^{* * *}$ \\
\hline
\end{tabular}

Values are presented as mean \pm standard deviation.

${ }^{*} p<0.05,{ }^{* \star} p<0.01,{ }^{* * *} p<0.001$.

PTSD, post-traumatic stress disorder; AD, adjustment disorder; BDI, Beck Depression Inventory; MMPI-2, Minnesota Multiphasic Personality Inventory 2; L, lie; K, correction; S, superlative self-presentation; HS, hypochondriasis; D, depression; HY, hysteria; PD, psychopathic deviate; MF, masculinity-femininity; PA, paranoia; PT, psychasthenia; SC, schizophrenia; MA, hypomania; SI, social introversion. 


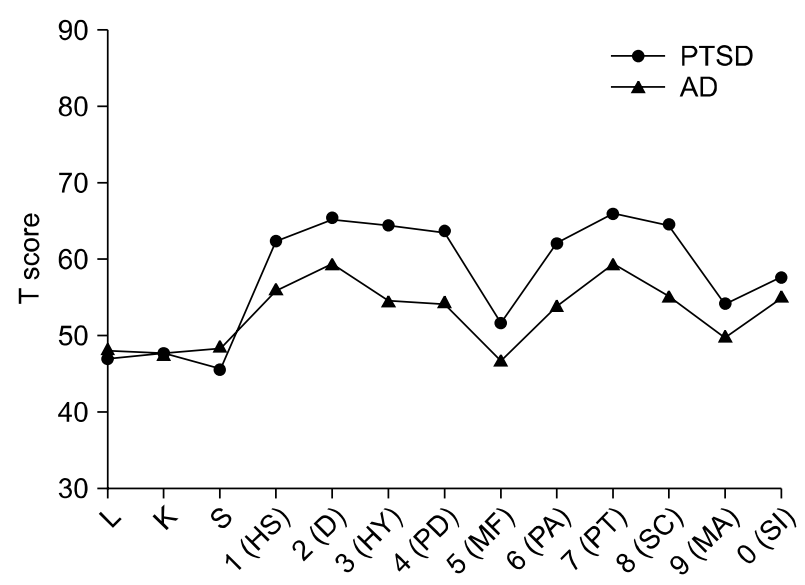

Fig. 1. Illustration of Minnesota Multiphasic Personality Inventory (MMPI-2) results between post-traumatic stress disorder (PTSD) and adjustment disorder (AD) group.

HS, hypochondriasis; D, depression; HY, hysteria; PD, psychopathic deviate; MF, masculinity-femininity; PA, paranoia; PT, psychasthenia; SC, schizophrenia; MA, hypomania; SI, social introversion; L, lie; K, correction; S, superlative self-presentation.

ability, we conducted an analysis of covariance (ANCOVA) with group as an independent variable and memory functions as dependent variables after controlling depressive symptoms (covariate).

\section{RESULTS}

\section{Demographic Characteristics}

There were no significant differences between people with PTSD and people with AD in gender, age, education, estimated IQ, and elapsed time after accident (Table 1).

\section{Memory Tests and BDI}

The results of the t-tests are presented in Table 2. The participants with AD had significantly higher scores than the participants with PTSD in immediate verbal memory, $t(45)=-3.20, p<0.01$, and delayed verbal recognition, $t(45)=-3.59, p<0.01$. The difference in delayed verbal recall was marginally significant, $t(45)=-1.99, p=0.053$. There was no difference for immediate figure recall, $t(45)=-0.38, p=0.70$, or delayed figure recognition, $t(45)=-0.48, p=0.64$, between groups.

PTSD group showed more severe depressive symptoms than AD group (34.56 vs. 21.35 respectively), $t(46)=4.71$, $p<0.001$. Correlation analysis showed that the severity of depressive symptom was significantly correlated with immediate verbal recall, $r=-0.56, p<0.001$; delayed recall, $r=-0.38, p<0.05$; delayed recognition, $r=-0.55, p<$ 0.001 ; and the PK scale, $r=0.56, p<0.001$. No significant differences were found in the ANCOVA for immediate verbal recall, $F(1,45)=1.78, p=0.19$; delayed recall, $F(1,45)=0.45, p=0.51$; and delayed recognition $F(1,45)=$ $3.07, p=0.08$; respectively.

\section{MMPI-2}

The scales of the MMPI-2 that differed significantly across groups were HS, $t(47)=2.09, p<0.05$; HY, $t(47)=3.12, \quad p<0.01 ; \mathrm{PD}, t(46)=2.68, p<0.05 ;$ PA, $t(47)=2.05, p<0.05$; SC, $t(47)=2.44, p<0.05$; and PK, $t(47)=2.20, p<0.05$. Results indicated that scales D and PT had the highest scores in the group with PTSD, but these scales did not differ across groups. The mean MMPI-2 profile of group with PTSD and group with AD are presented in Fig. 1.

\section{DISCUSSION}

The purpose of this study was to explore tools for the differential diagnosis of PTSD and AD groups after a vehicle accident. We compared verbal and visual memory, MMPI-2 scales, and BDI of two groups and found that verbal memory tests and MMPI-2 sub-scales can be used to differentiate the two groups.

First, we found that the group with AD performed significantly better than the group with PTSD in immediate verbal memory, delayed verbal recall, and delayed verbal recognition. In our study, the difference between groups could not be explained by differences in estimated intelligence, education level, or time since the traffic accident. These results are in accord with previous studies that demonstrated differences in verbal memory ability between participants with PTSD and Non-PTSD. ${ }^{14,18,42)}$

Our results suggest that people with PTSD have more difficulty in the encoding, processing and organization of verbal information than people with AD. However, further analysis indicated that verbal memory impairment in participants with PTSD was significantly influenced by depressive symptoms. These results are also in line with previous reports. ${ }^{18,42,44)}$ Another possibility for this result is that the interaction of PTSD and depressive symptoms may have a negative influence on verbal memory ability. $^{42)}$

Several studies have demonstrated an independent effect of PTSD symptoms on memory impairment. But, many studies included subjects who were diagnosed with anxiety and depressive disorders after traffic accidents, which complicate the process of determining the actual associations among PTSD symptoms, depressive symptoms 
and memory impairment. Thus, we included people with PTSD or AD but without a major depressive disorder diagnosis. To better understand the pathology of PTSD, it is important to explore the unique effect of PTSD symptoms and depressive symptoms on cognitive dysfunction in future.

In our study, there were no significant differences in immediate and delayed figure recall between the group of people with PTSD and the group of people with AD. Although a few studies have demonstrated differences in figure memory ability between individuals with PTSD and control groups, ${ }^{14,44)}$ other studies have reported no difference in figure memory ability between people with PTSD and people without PTSD. ${ }^{16,45,46)}$ Result of our study may be caused by measurement tool. We used Complex Figure Test of Rey-Kim Memory Test and this tool might be unsensitive differentiating visual memory ability between PTSD and AD. Considering this study and previous studies, difference of visual memory between PTSD and AD is unclear and more research is needed.

The results of our study revealed that the MMPI-2 clinical scales could be a useful index in the differentiation of PTSD and AD group. HS, HY, PD, PA, SC, and PK scores were significantly higher in participants with PTSD compared to the group with AD. Specially, considering previous studies, $t$-value and effect size of our study, powerful scale for differentiating two groups was the HY, SC, and PD. Higher scores on the HY scale may be reflect concentration problems, somatic complaints, helplessness, and anger control problems of PTSD. ${ }^{47,48)}$ Previous studies reported that SC scale of the MMPI-2 was important for the diagnosis of PTSD. ${ }^{25,26)} \mathrm{SC}$ scale can be reflect the problem of self-integration after trauma experience. And, social and inner alienation of individual with PTSD may be reflected in PD scale.

The PK scale also had good discriminative sensitivity, consistent with previous studies, ${ }^{25)}$ which suggested that the PK scale increased the diagnostic accuracy of MMPI-2 in differentiating participants with PTSD from control groups and other clinical samples. Adkins et al. ${ }^{49)}$ demonstrated that the PK scale showed good test-retest reliability and internal consistency. In our study, the D-PT code type of MMPI-2 increased prominently compared to other scales in the PTSD group; however, two groups in our study did not exhibit statistically different scores on these two scales. This implies that these D and PT scales may be not sensitive enough to differentiate PTSD and AD group.

To date, studies about the differential diagnosis of groups of individuals with PTSD and groups of indivi- duals with AD are rather rare, and our study is a meaningful attempt to fill this gap in the literature. The differential diagnosis of these disorders is difficult because the subjective and objective symptoms of people with PTSD and people with AD are often ambiguous or vague. Thus, identifying indices for a differential diagnosis between PTSD and AD is absolutely necessary.

We think that PTSD patients show dysfunction of memory efficacy compared to adjustment disorder and other disorders (e.g., major depressive disorder, anxiety disorder) in clinical setting and PTSD patients with traffic accident report higher scores in PA, PT, SC, HS and HY scale of MMPI-2 than Non-PTSD group (including adjustment disorder). Especially, we suggest that about 10 point difference between PTSD and AD in HY, PD, and SC scale of MMPI-2 can be useful in differentiating two groups.

This study has several limitations. First, all the patients in our study were taking medication, which could have influenced the symptom severity of patients and thus bias our results. Second, the sample size in this study is relatively small, which could be attenuate generalization of our results. Thus, a larger sample of patients with PTSD will be necessary for future studies.

In summary, our results showed a significant decrease in immediate verbal memory, delayed verbal recognition, and delayed verbal recall in participants with PTSD compared to participants with AD. However, these verbal memory impairments were significantly influenced by the depressive symptoms of participants with PTSD. The scores on the HS, HY, PD, PA, SC, and PK scales of the MMPI-2 were significantly higher in the groups of participants with PTSD, and specially, HY, SC, and PD was powerful discriminative scale. Our results suggest that verbal memory abilities and MMPI-2 clinical scales, especially HY, SC, and PD could be useful tools for discriminating PTSD and AD.

\section{Acknowledgments}

This research was supported by the Basic Science Research Program through the National Research Foundation of Korea (NRF) funded by the Ministry of Education, Science and Technology (No. 2012R1A1A2043992).

\section{REFERENCES}

1. American Psychiatric Association (APA). Diagnostic and statistical manual of mental disorders, 4th edition revised. Washington DC: American Psychiatric Association, 2000.

2. Alim TN, Feder A, Graves RE, Wang Y, Weaver J, Westphal $\mathrm{M}$, et al. Trauma, resilience, and recovery in a 
high-risk African-American population. Am J Psychiatry 2008;165:1566-1575.

3. Blanchard EB, Hickling EJ, Mitnick N, Taylor AE, Loos WR, Buckley TC. The impact of severity of physical injury and perception of life threat in the development of posttraumatic stress disorder in motor vehicle accident victims. Behav Res Ther 1995;33:529-534.

4. Brewin CR, Andrews B, Valentine JD. Meta-analysis of risk factors for posttraumatic stress disorder in trauma-exposed adults. J Consult Clin Psychol 2000;68:748-766.

5. Dougall AL, Ursano RJ, Posluszny DM, Fullerton CS, Baum A. Predictors of posttraumatic stress among victims of motor vehicle accidents. Psychosom Med 2001;63:402411.

6. Gil S. Coping style in predicting posttraumatic stress disorder among Israeli students. Anxiety Stress Copin 2005;18: 351-359.

7. Ozer EJ, Best SR, Lipsey TL, Weiss DS. Predictors of posttraumatic stress disorder and symptoms in adults: a meta-analysis. Psychol Bull 2003;129:52-73.

8. Pietrzak RH, Johnson DC, Goldstein MB, Malley JC, Southwick SM. Psychological resilience and postdeployment social support protect against traumatic stress and depressive symptoms in soldiers returning from Operations Enduring Freedom and Iraqi Freedom. Depress Anxiety 2009; 26:745-751.

9. Stallard P, Smith E. Appraisals and cognitive coping styles associated with chronic post-traumatic symptoms in child road traffic accident survivors. J Child Psychol Psychiatry 2007;48:194-201.

10. Vogt DS, Tanner LR. Risk and resilience factors for posttraumatic stress symptomatology in Gulf War I veterans. J Trauma Stress 2007;20:27-38.

11. Bremner JD, Randall P, Scott TM, Capelli S, Delaney R, McCarthy G, et al. Deficits in short-term memory in adult survivors of childhood abuse. Psychiatry Res 1995;59:97107.

12. Bremner JD, Scott TM, Delaney RC, Southwick SM, Mason JW, Johnson DR, et al. Deficits in short-term memory in posttraumatic stress disorder. Am J Psychiatry 1993;150: 1015-1019.

13. Jelinek L, Jacobsen D, Kellner M, Larbig F, Biesold KH, Barre $\mathrm{K}$, et al. Verbal and nonverbal memory functioning in posttraumatic stress disorder (PTSD). J Clin Exp Neuropsychol 2006;28:940-948.

14. Parslow RA, Jorm AF. Pretrauma and posttrauma neurocognitive functioning and PTSD symptoms in a community sample of young adults. Am J Psychiatry 2007;164:509-515.

15. Bremner JD, Vermetten E, Afzal N, Vythilingam M. Deficits in verbal declarative memory function in women with childhood sexual abuse-related posttraumatic stress disorder. J Nerv Ment Dis 2004;192:643-649.

16. Geuze E, Vermetten E, de Kloet CS, Hijman R, Westenberg HG. Neuropsychological performance is related to current social and occupational functioning in veterans with posttraumatic stress disorder. Depress Anxiety 2009;26:715.

17. Johnsen GE, Kanagaratnam P, Asbjørnsen AE. Memory impairments in posttraumatic stress disorder are related to depression. J Anxiety Disord 2008;22:464-474.

18. Johnsen GE, Asbjørnsen AE. Verbal learning and memory impairments in posttraumatic stress disorder: the role of encoding strategies. Psychiatry Res 2009;165:68-77.

19. Burt DB, Zembar MJ, Niederehe G. Depression and memory impairment: a meta-analysis of the association, its pattern, and specificity. Psychol Bull 1995;117:285-305.

20. Gilbertson MW, Gurvits TV, Lasko NB, Orr SP, Pitman RK. Multivariate assessment of explicit memory function in combat veterans with posttraumatic stress disorder. J Trauma Stress 2001;14:413-432.

21. Burriss L, Ayers E, Ginsberg J, Powell DA. Learning and memory impairment in PTSD: relationship to depression. Depress Anxiety 2008;25:149-157.

22. Forbes D, Creamer M, McHugh T. MMPI-2 data for Australian Vietnam Veterans with combat-related PTSD. J Trauma Stress 1999;12:371-378.

23. Greenblatt RL, Davis WE. Differential diagnosis of PTSD, schizophrenia, and depression with the MMPI-2. J Clin Psychol 1999;55:217-223.

24. Munley PH, Bains DS, Bloem WD, Busby RM. Post-traumatic stress disorder and the MMPI-2. J Trauma Stress 1995;8:171-178.

25. Lyons JA, Wheeler-Cox T. MMPI, MMPI-2 and PTSD: overview of scores, scales, and profiles. J Trauma Stress 1999;12:175-183.

26. Rademaker AR, Kleber RJ, Meijer ME, Vermetten E. Investigating the MMPI-2 trauma profile in treatmentseeking peacekeepers. J Pers Assess 2009;91:593-600.

27. Scott RL, Knoth RL, Beltran-Quiones M, Gomez N. Assessment of psychological functioning in adolescent earthquake victims in Colombia using the MMPI-A. J Trauma Stress 2003;16:49-57.

28. Glenn DM, Beckham JC, Sampson WS, Feldman ME, Hertzberg MA, Moore SD. MMPI-2 profiles of Gulf and Vietnam combat veterans with chronic posttraumatic stress disorder. J Clin Psychol 2002;58:371-381.

29. Weyermann AG, Norris FH, Hyer LA. Examining comorbidity and posttraumatic stress disorder in a Vietnam veteran population using the MMPI-2. J Trauma Stress 1996;9:353-360.

30. Wilson JP, Walker AJ. Toward an MMPI trauma profile. $J$ Trauma Stress 1990;3:151-168.

31. Wise EA. Diagnosing posttraumatic stress disorder with the MMPI clinical scales: A review of the literature. J Psychopathol Behav Assess 1996;18:71-82.

32. Forbes D, Creamer M, Allen N, McHugh T, Debenham P, Hopwood M. MMPI-2 as a predictor of change in PTSD symptom clusters: a further analysis of the Forbes et al. (2002) data set. J Pers Assess 2003;81:183-186.

33. Keane TM, Malloy PF, Fairbank JA. Empirical development of an MMPI subscale for the assessment of combat-related posttraumatic stress disorder. J Consult Clin Psychol 1984; 52:888-891.

34. Yeom TH, Park YS, Oh KJ, Kim JK, Lee YH. K-WAIS manual. Seoul: Korea Guidance, 1992.

35. Doppelt JE. Estimating the full scale score on the Wechsler adult intelligence scale from scores on four subtests. $J$ Consult Psychol 1956;20:63-66.

36. Silverstein AB. Two- and four-subtest short forms of the Wechsler Adult Intelligence Scale-Revised. J Counslt Clin Psychol 1982;50:415-418.

37. Kim HG. Rey-Kim Memory Test Manual. Daegu: Neuropsychology Press, 1999.

38. Hathaway S, McKinley JC. Minnesota Multiphasic Personality Inventory 2 (MMPI-2). Columbus, OH: Merrill Prentice-Hall; 1989.

39. Han OS, Hong JP. SCID-I (Structured Clinical Interview for DSM-IV): Korean Version. Seoul: Hana Medicine Press, 2000.

40. Beck AT, Ward CH, Mendelson M, Mock J, Erbaugh J. An 
inventory for measuring depression. Arch Gen Psychiatry 1961;4:561-571.

41. Lee MK, Lee YH, Jung HY, Choi JH, Kim SH, Kim YG, et al. A standardization study of beck depression inventory 2-Korean Version (K-BDI). Korean J Psychopathol 1995; 4:96-104.

42. Johnsen GE, Asbjørnsen AE. Consistent impaired verbal memory in PTSD: a meta-analysis. J Affect Disord 2008; 111:74-82.

43. Brandes D, Ben-Schachar G, Gilboa A, Bonne O, Freedman $\mathrm{S}$, Shalev AY. PTSD symptoms and cognitive performance in recent trauma survivors. Psychiatry Res 2002;110:231238.

44. Uddo M, Vasterling JJ, Brailey K, Sutker PB. Memory and attention in combat-related post-traumatic stress disorder (PTSD). J Psychopathol Behav Assess 1993;15:43-52.

45. Neylan TC, Lenoci M, Rothlind J, Metzler TJ, Schuff N,
$\mathrm{Du} \mathrm{AT}$, et al. Attention, learning, and memory in posttraumatic stress disorder. J Trauma Stress 2004;17:41-46.

46. Stein MB, Kennedy CM, Twamley EW. Neuropsychological function in female victims of intimate partner violence with and without posttraumatic stress disorder. Biol Psychiatry 2002;52:1079-1088.

47. Kim ZS. Multiphasic Personality Inventory. Seoul: Seoul National University Press, 2001.

48. James N, Butcher JR, Graham YS, Ben-Porath A, Tellegen W, Grant D, et al. Minnesota Multiphasic Personality Inventory-2 (MMPI-2) Manual. Minneapolis, MN: University of Minnesota Press; 2001.

49. Adkins JW, Weathers FW, McDevitt-Murphy M, Daniels JB. Psychometric properties of seven self-report measures of posttraumatic stress disorder in college students with mixed civilian trauma exposure. J Anxiety Disord 2008;22:13931402. 